\title{
Perfil bucodental y factores asociados a caries en niños de la ciudad de Bogotá
}

\section{Resumen}

Objetivo: Determinar el perfil epidemiológico bucodental y los factores relacionados con hábitos de alimentación y de higiene oral, asociados a caries, en niños de 2 a 4 años afiliados a la EPS Sanitas, en la ciudad de Bogotá. Material y Métodos: Se realizó un estudio descriptivo de corte transversal con aplicación de un instrumento para evaluar el perfil bucodental y los hábitos de higiene y de alimentación de cada paciente. Los evaluadores fueron especialistas en Odontopediatría, y para cada caso se firmó consentimiento informado. Resultados: se evaluó un total de 352 niños. El 58,2\% no presenta caries, el 73\% no presentaba obturaciones, y el $94 \%$ no tenía dientes extraídos. Se documentó que el 29,5\% de los niños presentaban lesiones de mancha blanca. La prevalencia
María Carolina-Ruiz ${ }^{1}$

María Camila-Amador ${ }^{2}$

Piedad-Carrillo ${ }^{3}$

\section{Artigo Original}

\section{Perfil bucodental e fatores associados á cárie em crianças na cidade de Bogotá}

\section{Resumo}

Objetivo: Determinar o perfil epidemiológico bucodental e os fatores relacionados com os há- de caries en los niños de 2 años fue del 37,36\% y para los niños de 3 y 4 años fue del 40,87\% y del $55,55 \%$ respectivamente. El índice ceo total fue de 2,51. El $73 \%$ de los niños se cepilla menos de 3 veces al día, el 38,2\% manifiesta que nadie suministra educación en salud oral y el $47 \%$ consume alimentos en la noche después del último cepillado. No se documentó asociación estadística entre la presencia de caries y las variables incluidas en los hábitos de higiene y de alimentación que se estudiaron. Conclusiones: Se recomienda fortalecer la educación a niños y cuidadores relacionada con los hábitos de higiene oral y de alimentación y realizar nuevos estudios para evaluar el impacto de las medidas implementadas.

Palabras clave: caries dental, epidemiología, hábitos, preescolares, prevalencia, higiene bucal.

\footnotetext{
${ }^{1}$ Estomatóloga Pediatra Universidad Nacional de Colombia.

${ }^{2}$ Odontopediatra Pontificia Universidad Javeriana.

${ }^{3}$ Médico Epidemióloga Universidad del Rosario. Subgerente Médico Regional. Organización Sanitas Internacional.
} 
com aplicação de um instrumento para avaliar o perfil bucodental e os hábitos de higiene e de alimentação de cada paciente. Os avaliadores eram especialistas em Odontopediatria, e para cada caso um termo de consentimento informado foi assinado. Resultados: Foram avaliadas 352 crianças - 58.2\% não apresentavam cárie; $73 \%$ não apresentavam restaurações, e $94 \%$ não tinham dentes extraídos. Foi observado que 29.5\% das crianças apresentavam lesões de mancha branca. A prevalência de cárie em crianças de 2 anos foi de $37,36 \%$, para crianças de 3 e 4 anos foi de $40,87 \%$ e de $55,55 \%$ respectivamente. O índice ceo total foi $2,51.73 \%$ das crianças escova os dentes menos de 3 vezes ao dia, 38,2\% manifesta que ninguém realiza ações de educação sobre saúde bucal, e $47 \%$ consome alimentos à noite depois da última escovada. Não se documentou associação estatística entre a presença de cárie e as variáveis estudadas sobre hábitos de higiene e alimentação. Conclusões: É recomendado fortalecer a educação e os cuidados relacionados aos hábitos de higiene bucal e de alimentação das crianças, e também realizar novos estudos para avaliar o impacto das medidas implementadas.

Palabras chave: cárie, epidemiologia, hábitos, prevalência, higiene oral.

\section{Original article}

\section{Orodental profile and associated factors with oral caries in childrens of Bogotá}

\begin{abstract}
Objective: To determine the oral epidemiological profile and the factors related to eating habits and oral hygiene associated with caries, in children from 2 to 4 years, members of the EPS Sanitas in Bogotá. Material and methods: A cross sectional study was made, with application of an instrument to evaluate the profile and the habits of hygiene and diet of each patient. The evaluators were specialists in Pediatric Dentistry, and in each case informed consent was signed. Results: 352 children were evaluated. $58.2 \%$ had no caries, $73 \%$ had no fillings, and $94 \%$ had teeth extracted. It was reported that $29.5 \%$ of the children had white spot lesions. The prevalence of caries in children aged
\end{abstract}

2 years was $37.36 \%$ and for children with 3 and 4 years the prevalence was $40.87 \%$ and $55.55 \%$ respectively. The ceo-d total index was 2.51 . $73 \%$ of the children brush their teeth less than 3 times a day, $38.2 \%$ say that no one provides oral health education and $47 \%$ consume food in the evening after the last brushing. No statistical association was documented between the presence of caries and the other variables included. Conclusions: It is recommended to strengthen education to children and caregivers related to oral hygiene and feeding and to realize further studies to assess the impact of measures implemented.

Key words: dental caries, epidemiology, habits, child, preschool, prevalence, oral hygiene 


\section{Introducción}

La caries dental continúa siendo uno de los principales problemas de la salud pública en odontología, siendo la caries de infancia temprana, una de las más agresivas y difíciles de tratar. Este tipo de caries se caracteriza por la presencia de uno o más dientes cariados, perdidos o superficies dentales obturadas en niños de 71 meses de edad o menores, aclarando que cualquier signo de caries dental en niños menores de 3 años se define como caries de infancia severa. (1) La primera evidencia clínica es la presencia de lesiones de mancha blanca en los dientes anteriores superiores temporales que pueden avanzar hasta un estadio de cavitación que si no es tratado a tiempo lleva a grades destrucciones de la estructura del coronal; los otros dientes temporales pueden o no estar involucrados dependiendo del tiempo que estén con el factor etiológico. $(2,3,4,5,6,7)$ El factor etiológico está determinado por cuatro factores que son paciente, sustrato, microorganismos y tiempo este ultimo el que determinara la agresividad de la caries. $(8,9,10,11,12,13,14)$ Teniendo en cuenta la integralidad del niño las secuelas de la caries de infancia temprana no están solamente asociadas a la parte dental, sino que van a estar relacionadas con el aspecto sistémico, psicológico, de fonación y de autoestima que no permitirán un desarrollo normal del niño en su entorno cultural y social. $(15,16,17,18)$

Con el fin de evaluar el estado de la salud oral de la población colombiana, aproximadamente cada 10 años se realiza en nuestro país un Estudio Nacional de Salud Bucal (ENSAB) (19). De acuerdo con los resultados encontrados en el tercer ENSAB, el cual fue realizado en el año de 1998, en los niños de 5 años se encontró que el $60.4 \%$ tenía historia de caries y el índice ceo-d fue de 3.0 para esta edad. Al comparar los resul- tados de este estudio con los obtenidos durante el ENSAB previo, realizado en el año de 1977, se observa una reducción del $30 \%$ en el número promedio de dientes con historia de caries, y el índice ceo-d paso de 4.2 en la encuesta realizada entre 1977-80 a 3.0 en el ENSAB de 1998. Aunque el país no alcanzó la meta OMS / FDI (20) de salud bucodental para este grupo de población, el cual es de 2, el hecho de que el 39.6\% de estos niños estén sin historia de caries, muestra un impacto moderado de los niveles de salud en la dentición primaria.

Desde entonces, en la ciudad de Bogotá se han realizado diversos estudios locales a nivel de hospitales y de algunas localidades, los cuales muestran una frecuencia de caries en niños menores de 5 años, que varía entre el 22 y el 51\%, y de igual manera, se evidencian alteraciones en los hábitos alimentarios y de higiene oral, los cuales se asocian a la aparición de caries. (21)

Debido a que no hay datos recientes que permitan tener una aproximación de la prevalencia de caries en niños de 2 a 4 años en la ciudad de Bogotá, se planteó la necesidad de realizar un estudio con el fin de determinar el perfil bucodental, la prevalencia de caries, los hábitos de higiene y de alimentación que inciden en la aparición de caries en este grupo de edad, y la asociación de estos factores con la aparición de caries.

\section{Material y métodos}

Se realizó un estudio descriptivo de corte transversal, dirigido a la población de niños de 2, 3 y 4 años de edad afiliados a una compañía aseguradora de salud en la ciudad de Bogotá. El cálculo del tamaño de muestra se realizó utilizando datos de prevalencia de caries para cada uno de los grupos de edad estudiados, de acuerdo a lo referido en la literatura. El número de niños a 
incluir en el estudio se distribuyó en 5 sedes de prestación de servicio de Odontopediatría en la ciudad de Bogotá.

Los pacientes fueron evaluados por un total de 4 odontólogos con especialización en Odontopediatría, quienes fueron previamente estandarizados con relación a la evaluación clínica de los pacientes y a la metodología a seguir para el registro de la información. El instrumento de recolección de información utilizado fue evaluado en una prueba piloto y se encuentra dividido en 3 secciones: Evaluación Clínica del paciente, Hábitos de Alimentación y Hábitos de Higiene Oral. Para la información relacionada con la evaluación clínica se tuvieron en cuenta las recomendaciones establecidas por la OMS (22) con relación a la información a recopilar para elaborar un Perfil Epidemiológico Bucodental, y se procedió al cálculo del índice ceo global y para cada grupo de edad. Con relación a los hábitos de alimentación y de higiene oral se registró información relacionada con el uso de cepillo de dientes, de crema dental, frecuencia de cepillado al día, número de comidas principales al día, hábito de consumo de alimentos adicionales y consumo de alimentos posterior al último cepillado dental. Posteriormente se buscó asociación entre las distintas variables con la presencia de caries. Para realizar la inclusión de los casos se procedió a explicar los objetivos del estudio al acompañante adulto del paciente y se le solicitó firmar el respectivo consentimiento informado. La selección de los niños a incluir en el estudio se realizó mediante un muestreo aleatorio simple estratificado para cada uno de los grupos de edad. Los resultados de cada caso fueron registrados en una base de datos diseñada en Excel y los análisis estadísticos se realizaron utilizando el paquete estadístico STATA versión 8.0 El protocolo de investigación fue evaluado y aproba- do por el comité de ética de investigación institucional. Se obtuvo consentimiento por escrito del representante legal de cada paciente.

\section{Resultados}

Fueron incluidos en el estudio un total de 352 niños con edades comprendidas entre los 2 y los 4 años de edad, y para cada grupo de edad se logró alcanzar el tamaño de muestra esperado. No hubo diferencias importantes en cuanto a la distribución por sexo (47\% fueron niñas y 53\% fueron niños). En cuanto al nivel de escolaridad de las madres se encontró que el 59\% tenían una formación técnica o inferior, y el $41 \%$ restante tenían formación universitaria.

\section{Perfil Bucodental}

La Gingivitis fue el trastorno de la mucosa oral más observado, el cual fue documentado en el $23 \%$ de los niños. E1 $76 \%$ restante no presentaba lesiones de este tipo. El 80,1\% de los niños examinados no presenta lesiones relacionadas con opacidad e hipoplasia. En el grupo que presentaba estas lesiones se encontró que la opacidad delimitada y la hipoplasia son las alteraciones más frecuentes.

El 58,2\% de los niños examinados no presenta caries. En cuanto al grupo de niños con caries se encontró que 48 niños (33\%) tenía 1 sola caries, 29 niños $(19,7 \%)$ tenía 2 caries y los restantes tenían 3 o más caries. Se encontraron valores extremos de 12 y 13 caries en dos niños. La prevalencia de caries en los niños de 2 años fue del $37,36 \%$ y para los niños de 3 y 4 años fue del $40,87 \%$ y del 55,55\% respectivamente. El $73 \%$ de los niños no presentaba obturaciones, y el $94 \%$ no tenía dientes extraídos. Adicionalmente, se documentó que el 29,5\% de los niños exami- 
nados presentaban lesiones de mancha blanca.

La tabla 1 resume el cálculo del índice ceo para cada uno de los grupos de edad.

\section{Hábitos de higiene oral}

En cuanto a los hábitos de higiene oral, se documenta que el $73 \%$ de los niños se cepilla los

Tabla 1. Cálculo del índice ceo.

\begin{tabular}{|c|c|}
\hline Edad & Índice ceo \\
\hline 2 años & 1,96 \\
\hline 3 años & 3,00 \\
\hline 4 años & 3,13 \\
\hline Total general & 2,51 \\
\hline
\end{tabular}

Fuente: Base de datos de registro de información. dientes menos de 3 veces al día. El 95,6\% utiliza crema dental y en este grupo el 10,5\% utiliza crema sin flúor.

El 88,7\% de los niños iniciaron el hábito de cepillado después de los 6 meses de edad y en cuanto a los conocimientos relacionados con salud oral, estos son suministrados especialmente por el odontólogo $(47 \%)$, y se debe destacar que el $38,2 \%$ de los casos refiere que nadie le suministra información relacionada con el tema.

\section{Hábitos de alimentación}

Con relación a los hábitos de alimentación se documenta que el $47 \%$ de los niños consume algún alimento antes de dormir después de haberse cepillado los dientes en la noche, que el $92 \%$ de los niños consume 5 comidas principales

Tabla 2.

\begin{tabular}{|c|c|c|c|}
\hline Variable & $\begin{array}{c}\text { Caries } \\
\mathbf{n = 1 4 7} \\
\mathbf{n}(\%)\end{array}$ & $\begin{array}{c}\text { No caries } \\
\mathbf{n = 2 0 4} \\
\mathbf{n}(\%)\end{array}$ & Valor $\mathbf{p}$ \\
\hline $\begin{array}{c}\text { Consumo de otras comidas } \\
\text { adicionales a las principales }\end{array}$ & $107(72,8)$ & $131(64,2)$ & 0,09 \\
\hline $\begin{array}{c}\text { Come en la noche antes de } \\
\text { dormir }\end{array}$ & $76(57,1)$ & $90(44,1)$ & 0,16 \\
\hline $\begin{array}{c}\text { Edad inicio cepillado }>6 \\
\text { meses }\end{array}$ & $131(89,1)$ & $181(88,3)$ & 0,81 \\
\hline Uso de crema & $142(96,6)$ & $195(95,1)$ & 0,5 \\
\hline $\begin{array}{c}\text { Frecuencia de cepillado }>= \\
\text { 3 veces al día }\end{array}$ & $113(76,9)$ & $144(70,2)$ & 0,17 \\
\hline $\begin{array}{c}\text { Uso compartido del cepillo } \\
\text { de dientes }\end{array}$ & $1(0,7)$ & $2(0,1)$ & 0,5 \\
\hline $\begin{array}{c}\text { Escolaridad universitaria } \\
\text { de la madre }\end{array}$ & $90(61,2)$ & $118(57,6)$ & 0,77 \\
\hline
\end{tabular}

Fuente: Base de datos de registro de información 
en el día y que el 67,8\% de los niños consume alimentos adicionales a estas comidas.

El análisis bivariado entre las distintas variables y la presencia o no de caries no mostró resultados con significancia estadística. Estos resultados se muestran en la tabla 2.

\section{Discusión}

El índice ceo encontrado en este estudio documenta valores para los niños de 3 y de 4 años comparables a los encontrados en el Estudio Nacional de Salud Bucal ENSAB III de 1998 (23), aunque este estudio evalúa niños de 5 años únicamente. Dado que la prevalencia de caries aumenta con la edad de acuerdo con lo referenciado en la literatura, se podría pensar que el índice ceo de la población de niños de 5 años pertenecientes a la aseguradora podría ser mayor. Adicionalmente, se debe tener en cuenta que si se incluyeran criterios diagnósticos de caries mas sensibles el índice sería superior, ya que por ejemplo, en el presente estudio se encontraron lesiones de mancha blanca en un $29.55 \%$ de los niños, información que no fue tenida en cuenta para el cálculo del índice.

Lo anterior evidencia la necesidad de realizar una nueva ENSAB ya que la última se realizó en el año de 1998 y los datos actuales podrían no corresponder a la realidad actual.

El aumento significativo en el índice ceo que se observa entre los pacientes de 2 y de 3 años puede estar asociado al hecho de que la primera consulta no siempre se da antes de los 2 años, edad ideal para iniciar un proceso de educación a los padres y cuidadores, sino en edades posteriores. Se evidencia entonces la presentación temprana de caries, y por tal motivo se resalta la necesidad de un diagnóstico precoz y al mismo tiempo de desarrollar actividades terapéuticas y preventivas específicas.

En cuanto a los hábitos de higiene oral es de destacar que del $100 \%$ de padres o acudientes encuestados solo uno respondió no usar cepillo dental. Esto puede deberse a limitaciones del cuestionario en el cual por deseo social, los padres tienden a dar respuestas ideales aunque no sean reales. Con relación a la frecuencia diaria de cepillado se observa que el $73 \%$ lo hace menos de tres veces, lo cual implica la importancia de instaurar campañas educativas que lleven a mejorar los hábitos de higiene oral, las cuales sean implementadas en el hogar pero también en los centros de estudio de los niños ya que en estos lugares se pasa la mayor parte del día.

De igual manera, llama la atención que solo el $10.5 \%$ de los niños encuestados utiliza crema sin flúor, lo cual sugiere que hay falta de información acerca del riesgo de la ingesta de las cremas fluoradas a temprana edad.

Los resultados de la pregunta relacionada con el suministro de información acerca de la forma correcta de cepillado son comparables a los encontrados en el estudio realizado por la Dra. Chavarro en el año 2000 en Bogotá, (24) en cuanto al porcentaje de padres informados por el odontólogo, siendo en ambos estudios el mas alto (43.9 vs 47.58). En este estudio se encontró un alto porcentaje (38.18\%) de padres que refieren no haber recibido información acerca del correcto cepillado. Esto es preocupante teniendo en cuenta que la mayoría de nuestra población asiste periódicamente a citas de Pediatría y de Programas de Crecimiento y Desarrollo. Es de destacar que los resultados no hacen referencia a las campañas masivas de los medios de comunicación, lo cual sugiere que estas podrían no tener el impacto esperado en cuanto a educación 
en higiene oral.

En cuanto a los hábitos de alimentación cabe destacar el hecho de que un alto porcentaje de niños $(47 \%)$ consume alimentos antes de irse a dormir después del último cepillado en la noche, que más del $90 \%$ consumen 5 comidas principales al día y que adicionalmente consumen otros alimentos, con una frecuencia diaria de cepillado inferior a 3 veces en el $73 \%$.

La aparición de caries es un fenómeno multifactorial, y aunque el análisis bivariado y multivariado del presente estudio no mostraron asociaciones significativas que así lo demuestren, probablemente porque el tamaño de muestra no fue calculado con este propósito, se debe destacar la presencia de múltiples factores que podrían estar influyendo en la aparición de caries en la población estudiada.

Lo anterior demuestra que es necesario afrontar el problema desde distintos puntos de vista, relacionados tanto con la educación a los niños, a los padres y cuidadores, empleando distintos tipos de estrategias. Utilizando la información anterior se va a diseñar un programa de prevención dirigido a mujeres gestantes, cuyo propósito sea lograr una disminución en la prevalencia de caries y del índice ceo-d en la población infantil. Los resultados de este estudio servirán de línea de base para futuras comparaciones que se realicen tras la realización de otras intervenciones.

Adicionalmente, se encuentra que en Colombia son pocos los estudios publicados sobre salud oral realizados en este grupo de edad, por lo cual no se dispone de datos con los cuales comparar los resultados encontrados en este trabajo. Lo anterior evidencia la importancia de realizar más estudios de este tipo en este rango de edad.

\section{Agradecimientos}

Agradecemos a la Organización Sanitas Internacional por su apoyo durante el Estudio y a los pacientes que participaron en el presente estudio.

\section{Referencias}

1. American Academy of Pediatric Dentistry. Clinical guideline on infant oral health care. Pediatric Dentistry 2003; 25 (7):54

2. Kroll, R. Stone, J. Nocturnal Bottle-Feeding as a Contributory cause of Rampant Dental Caries in the infant and Young Child. Journal of Dentistry for Children. 1967; 1(34):454

3. Ripa, L. Nursing Caries: a comprehensive review. Pediatric Dentistry1988;10(4): 268

4. ICDAS INTERNATIONAL CARIES ASSESSMENT AND DETECTION SYSTEM. ICDAS Foundation 2010. Disponible en www.icdas.org

5. Olivar, A. Atención estomatológica al binomio madre-hijo. Colombia: Editorial Universidad Nacional de Colombia. Odontología; 2006.

6. Johnsen, D. Background comparisons of pre $31 / 2$ year old children with nursing caries in four practice settings. Pediatric Dentistry. 1984; 6(1):50.

7. Herman, H. Preventive dental care: The role of the pediatrician. Pediatrics.1987; 80(1):108.

8. Kotlow, L. Breast feeding: A cause of dental caries in children. Journal of Dentistry for Children. 1977; May-Jun:25.

9. Bouga-Paraskaki, A. The use of the Nursing Bottle. Journal of Dentistry for Children. 1973; July-August: 51 
10. Ekstrand, KR. Oclusal Caries: Pathology, Diagnosis and Logical Management. Dent Update 2001; 28: 380-387

11. Eronat, N. Eden, E. A comparative study of some influencing factor of rampant or nursing caries in preschool children. Pediatric Dentistry. 1992; 16:15

12. Mc. Donald. R. Odontología Pediátrica y del Adolescente. Argentina, Editorial Médica Panamericana. 1990

13. Bordoni N; Castillo R, Escobar, A; Odontología Pediátrica La salud bucal del niño y el adolescente en el mundo actual. $1^{\text {a }}$ ed. Buenos Aires: Editorial Médica Panamericana;2010

14. Ayhan, H. Influencing factors of nursing caries. The Journal of Clinical Pediatric Dentistry. 1996;20(4):313

15. Ott, T. Kummer, A. Premature loss of the maxillary primary incisors: Effect on speech production. Journal of Dentistry for Children. 1995; May-Jun: 173

16. Schwartz, S. A child's sleeping habit a cause of nursing caries. Journal of Dentistry for Children. 1993 ; Jan-Feb: 22.

17. Riekman, G. Effect of premature lost of primary maxillary incisors on speech. Pediatric Dentistry. 1985;7(2):119-122.

18. Koroluk, L. Parental perceptions of the effects of maxillary incisor extractions in children with nursing caries. Journal of Dentistry for Children. 1991;May-Jun: 233-236

19. Ministerio de Salud, República de Colombia. Centro Nacional de Consultoría. III Estudio Nacional de Salud Bucal ENSAB III Colombia. Bogotá; 1999. Disponible en http: / / onsb.udea.edu.co/site/images/pdf/ensab3.pdf

20. OMS / FDI Metas de Salud Bucal para el año 2000. Ginebra 1979.

21. Chavarro, I. Prevalencia de caries del lactante y posibles factores asociados en preescolares de instituciones de Bogota, Presentación de resultados. Revista Pediatría 2001; 36 (2). Disponible en www.encolombia.com/medicina/pediatria/ pedi36201-prevalencia.htm

22. OMS. Encuesta de Salud Bucodental métodos básicos. $4^{\mathrm{a}}$ ed. Ginebra. 1997. Disponible en http://apps.who.int/bookorders $/$ anglais $/$ detart1.jsp? sesslan $=1 \& \operatorname{codlan}=3 \& \operatorname{cod} \operatorname{col}=15 \& \operatorname{cod} c h=4275$

23. Ministerio de Salud, República de Colombia. Centro Nacional de Consultoría. III Estudio Nacional de Salud Bucal ENSAB III Colombia. Bogotá; 1999. Disponible en http: / / onsb.udea.edu.co/site/images/pdf/ensab3.pdf

24. Chavarro, I. Prevalencia de caries del lactante y posibles factores asociados en preescolares de instituciones de Bogotá, Presentación de resultados. Revista Pediatría 2001; 36 (2). Disponible en www.encolombia.com/medicina/pediatria/ pedi36201-prevalencia.htm

Recibido: 24-04-12

Aceptado: 30-04-12

Correspondencia: karoruiz1@hotmail.com 